\title{
ERRATUM
}

Takeo W. Tsutsui • Tomohiro Inaba • Larry W. Fisher

Pamela Gehron Robey • Takeki Tsutsui

\section{In vitro chromosome aberration tests using human dental pulp cells to detect the carcinogenic potential of chemical agents}

\section{Erratum to: Odontology (2006) 94:44-50}

In the article by T.W. Tsutsui et al. entitled "In vitro chromosome aberration tests using human dental pulp cells to detect the carcinogenic potential of chemical agents", which appeared in Odontology vol. 94, no. 1 (pp. 44-50), parts of Figs. 1 and 2 appeared incorrectly. The microphotographs in Fig. 1C, E, and $\mathrm{G}$ were reversed (inverted right-left and top-bottom). The labels (letters and other symbols) are correctly positioned. The microphotograph in Fig. 1D was inverted top-bottom, but the labels are correctly positioned. In Fig. $2 \mathrm{~F}$, the microphotograph should be rotated $90^{\circ}$ degrees counterclockwise and the magnification should be adjusted; the labels are correctly positioned. In the figures reproduced above, the ones on the left are shown as they appeared incorrectly. The figures on the right are shown as they should correctly appear.

The online version of the original article can be found at http://dx.doi.org/10.1007/s10266-006-0065-1

T.W. Tsutsui $\cdot$ T. Tsutsui $(\bowtie)$

Department of Pharmacology, The Nippon Dental University School of Life Dentistry at Tokyo, 1-9-20 Fujimi, Chiyoda-ku, Tokyo

102-8159, Japan

Tel. +81-3-3261-8771; Fax +81-3-3263-5452

e-mail: takeki@tokyo.ndu.ac.jp

T. Inaba

Oral and Maxillofacial Surgery, The Nippon Dental University

Hospital at Tokyo, Tokyo, Japan

L.W. Fisher · P.G. Robey

Craniofacial and Skeletal Diseases Branch, National Institute of

Dental and Craniofacial Research, National Institutes of Health,

Department of Health and Human Services, Bethesda, MD, USA 

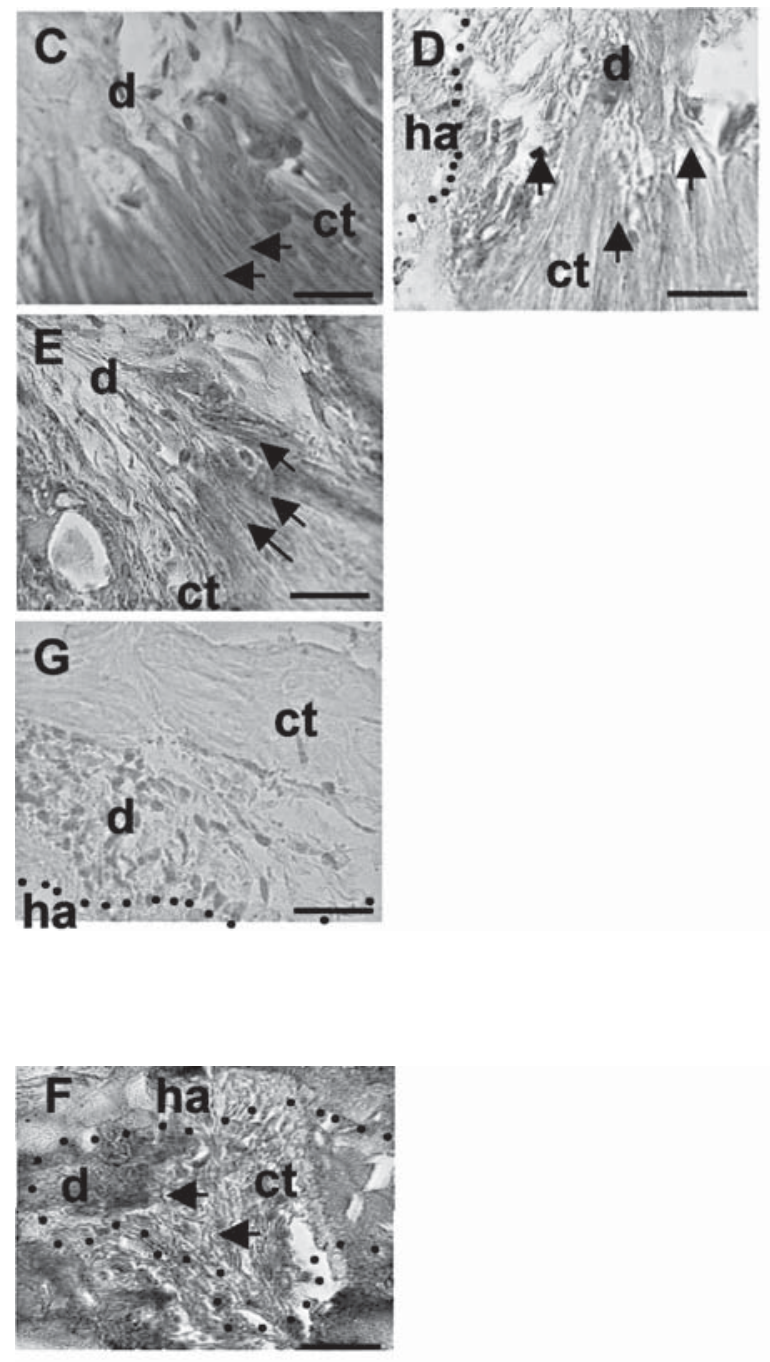

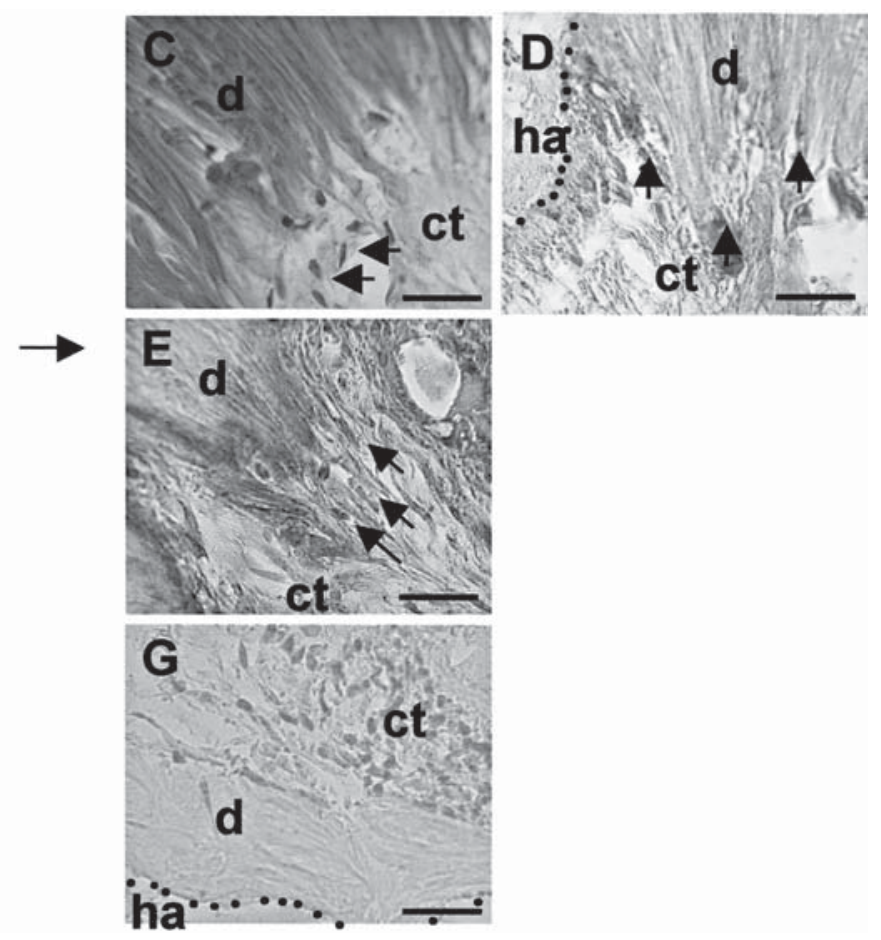

Fig. 1

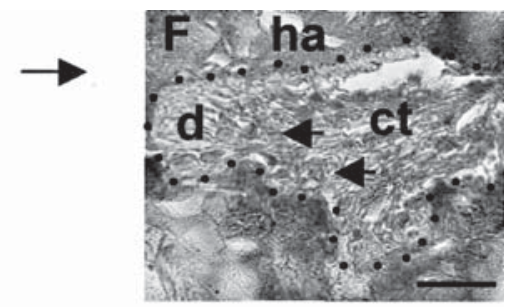

Fig. 2 